\title{
Phytosterol Recognition via Rationally Designed Molecularly Imprinted Polymers
}

\author{
Lachlan J. Schwarz ${ }^{\dagger}$, Brenda K. Y. Leung, Basil Danylec, Simon J. Harris, Reinhard I. Boysen (i) \\ and Milton T. W. Hearn * \\ Centre for Green Chemistry and Australian Centre for Research on Separation Science, School of Chemistry, \\ Monash University, Melbourne, VIC 3800, Australia; lachlan.schwarz@monash.edu (L.J.S.); \\ brenda.leung@monash.edu (B.K.Y.L.); basil.danylec@monash.edu (B.D.); \\ simon.harris@monash.edu (S.J.H.); reinhard.boysen@monash.edu (R.I.B.) \\ * Correspondence: milton.hearn@monash.edu; Tel.: +61-3-9905-4547 \\ † Current Address: School of Agricultural and Wine Sciences, Faculty of Science, Charles Sturt University, \\ Wagga Wagga, NSW 2678, Australia.
}

Received: 29 December 2017; Accepted: 4 February 2018; Published: 12 February 2018

\begin{abstract}
Molecularly imprinted polymers (MIPs) prepared via a semi-covalent imprinting strategy using stigmasteryl methacrylate as a polymerisable template have been evaluated by static binding methods for their ability to selectively capture other valuable phytosterol targets, including campesterol and brassicasterol. Design criteria based on molecular modelling procedures and interaction energy calculations were employed to aid the selection of the co-monomer type, as well as the choice of co-monomer:template ratios for the formation of the pre-polymerisation complex. These novel hybrid semi-covalently imprinted polymers employed $N, N^{\prime}$-dimethylacryl-amide $\left(N, N^{\prime}\right.$-DMAAM) as the functional co-monomer and displayed specific binding capacities in the range 5.2-5.9 $\mathrm{mg}$ sterol/g MIP resin. Their binding attributes and selectivities towards phytosterol compounds were significantly different to the corresponding MIPs prepared via non-covalent procedures or when compared to non-imprinted polymers. Cross-reactivity studies using stigmasterol, ergosterol, cholesterol, campesterol, and brassicasterol as single analytes revealed the importance of the A-ring C-3- $\beta$-hydroxyl group and the orientational preferences of the D-ring alkyl chain structures in their interaction in the templated cavity with the $N, N^{\prime}$-dimethylamide functional groups of the MIP. Finally, to obtain useful quantities of both campersterol and brassicasterol for these investigations, improved synthetic routes have been developed to permit the conversion of the more abundant, lower cost stigmasterol via a reactive aldehyde intermediate to these other sterols.
\end{abstract}

Keywords: phytosterols; molecular modelling; interaction energies; molecularly imprinted polymers; selectivity

\section{Introduction}

Phytosterols are naturally occurring bioactive compounds, which have attracted wide-spread interest in recent years within the biomedical research and pharmaceutical/nutraceutical communities due largely for their ability to (i) lower blood serum levels of LDL-cholesterol; (ii) inhibit oxidative stress and cellular deterioration; (iii) act as anti-inflammatory compounds; and (iv) for their role as steroidal intermediates and precursors in the production of several pharmaceuticals [1-3]. Although feasible, the production of phytosterols by total chemical synthesis methods is both technically challenging and expensive. Further, current methods for sourcing these compounds from plant extracts typically involve multi-step procedures based on a combination of distillation, liquid-liquid or supercritical fluid extractions, and crystallization methods. Often, these procedures lead to products 
in relatively low yields or poor quality [2,4-6]. An opportunity exists to explore alternative, more cost-effective technologies of lower energy consumption for the separation and purification of these valuable compounds from natural sources, including food and oil processing waste streams. In this context, molecularly imprinted polymers (MIPs) represent an attractive option for the isolation of phytosterols, since these functional polymers have been employed for other classes of compounds as re-usable adsorbents in solid-phase tank batch extraction procedures or packed-bed chromatographic formats [7-9].

MIPs are porous materials, designed to contain complimentary 'receptor-like' binding sites capable of recognising a specific molecular template [10]. Such MIPs can exhibit a 'molecular memory' for the template or structurally related molecular analogues [11-13]. To date, most studies involving sterol-related molecularly imprinted polymers, usually involving non-covalent self-assembly approaches, have focused on cholesterol [14-23] or estradiol [24-27]. The focus of this investigation was to explore an alternative method for the generation of a covalently imprinted MIP based on the use of the cleavable stigmasterol methacrylate as template, in silico determination of optimal co-monomer-template ratios, batch binding studies with several sterol analogues, and molecular modeling of the binding interactions. The studies reported herein document an alternative way to design and develop phytosterol-selective MIP adsorbents with the potential for more generic applications.

\section{Results and Discussion}

In this investigation, stigmasterol was utilised as a suitable molecular template due to its relative abundance and affordability, and also due to its capability to act as a common precursor for the synthesis of significantly more valuable sterols, such as campesterol and brassicasterol (Schemes 1-3). Molecularly imprinted polymers are often prepared by a self-assembly approach, wherein the molecular template and functional monomers are allowed to self-assemble in solution prior to polymerisation. The advantage of this approach is its technical simplicity. However, when attempts to make stigmasterol-imprinted polymers via self-assembly approaches with $N, N^{\prime}$-dimethylacrylamide ( $N, N^{\prime}$-DMAAM), methacrylic acid (MAA), or 4-vinylpyridine (4-VP) were initially pursued, upon evaluation of their binding properties, the derived polymers failed to demonstrate any significant imprinting effect for stigmasterol when compared to their corresponding non-imprinted control counterparts. The observed binding was largely associated with non-specific interactions. These studies confirmed an earlier report on the failure of the monomers MAA and 4-VP to generate by non-covalent self-assembly approaches stigmasterol-imprinted polymers with acceptable imprinting factors [28]. This outcome can be attributed to an inability to form a stable pre-polymerisation complex, involving the functional monomers and the sterol template, owing to the presence of only a single A-ring C-3 $\beta$-hydroxyl group of the sterol capable of forming a hydrogen bond with, for example, the $N, N^{\prime}$-dimethylamido group of the $N, N^{\prime}$-DMAAM monomer. Thermal disruption of this hydrogen bond during polymerisation would account for the low specific binding levels of the derived polymer. Analogous experiences have been reported for cholesterol as the template with self-assembling imprinting procedures $[17,20]$. Therefore, an alternative strategy was adopted based on the use of a semi-covalent MIP approach with the functional monomer and template combined via a formal covalent linkage.

\subsection{Selection of the Molecular Template}

Upon polymerisation with $N, N^{\prime}$-dimethylacrylamide ( $N, N^{\prime}$-DMAAM) as the co-monomer, the molecular template, stigmasteryl-3-O-methacrylate, generated a MIP which can be hydrolyzed to afford a complimentary cavity housing carboxylic acid and $N, N^{\prime}$-dimethylamido group functionalities as hydrogen bonding recognition units $[17,28]$. On the basis of molecular modelling investigations, it was anticipated that this MIP would be able to recognize not only stigmasterol but also the more valuable campesterol and brassicasterol. It was thus envisaged that the use of the more abundant and 
affordable stigmasterol would lead to a more cost-effective development of MIP technologies capable of recognizing similar steryl core backbones for both analytical and industrial-scale applications.

\subsection{Molecularly Imprinted Polymer Design}

For MIPs prepared by non-covalent methods, the design procedures typically involve in silico modelling of the self-assembled pre-polymerisation complexes, which aids in the selection of suitable functional monomers and the determination of appropriate template to monomer ratios $[13,29]$. In this current investigation, this modelling approach has been adapted in a novel three-stage manner to suit the design of hybrid MIPs, accommodating imprinted sites prepared via a semi-covalent methodology (i.e., using a polymerisable template) in combination with a co-monomer that can interact through both hydrogen bonding and other non-covalent processes. As a first step in this in silico modelling procedure, the impact of solvent was excluded in order to reduce the complexity of the system and the computation time. This step was achieved by conducting in silico modelling titrations of the stigmasteryl methacrylate functional template with increasing equivalents of the different co-monomers in order to form 'virtual' pre-polymerisation complexes using semi-empirical equilibrium geometry calculations based on a parameterized model number 3 (PM3) force field to derive the heat of formation values $\left(\Delta H_{\mathrm{f}}\right)$ and the energy of interaction $\left(\Delta E_{\mathrm{i}}\right)$ of the template, the co-monomer clusters, and the template:co-monomer cluster complexes. As a second step, all polymerisable atoms were then 'frozen' to mimic polymerisation using the Merck Molecular Force Field (MMFF) procedures, permitting any free monomer units to be excluded but including solvent and performing a 'virtual' base hydrolysis of the ester linkage, whereby the covalent bond between the steryl moiety and the methacrylate was cleaved. This step resulted in the ester being replaced by a carboxylic acid functionality. Finally, as a third step, stigmasterol was then re-introduced into the cavity and semi-empirical PM3 equilibrium geometry calculations performed again, including all of the solvated 'frozen' polymethacrylic acid units. This rebinding of stigmasterol to these 'virtual' cavities (Figure 1) revealed that the use of 2 mole equivalents of the functional co-monomer $N, N^{\prime}$-DMAAM resulted in silico in the thermodynamically most stable association, with a $\Delta E_{\mathrm{i}}$ of $-37.1 \mathrm{KJ} / \mathrm{mol}$. For comparative purposes, a non-covalent titration (at 2 monomer equivalents) was also conducted and revealed much smaller in silico-generated interaction energies $\left(\Delta E_{\mathrm{i}}\right.$ values $\left.<-5.0 \mathrm{~kJ} / \mathrm{mol}\right)$ for the same monomer type when employing a purely non-covalent self-assembly approach. In order to investigate the extent to which non-specific binding may contribute to the observed interaction energies, non-imprinted polymer systems were also modelled.

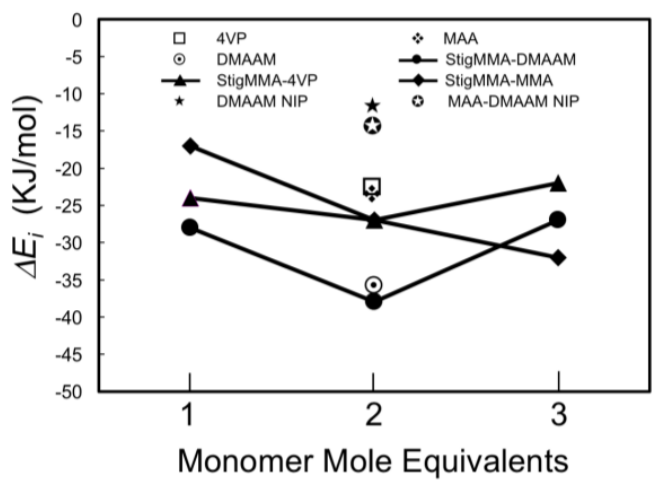

Figure 1. Modelling titration simulations for stigmasterol against three commercially available functional monomers, namely 4-vinylpyridine (4VP), methacrylic acid (MAA), and $N, N^{\prime}$-dimethylacrylamide $\left(N, N^{\prime}\right.$-DMAAM), with the change in interaction energy $\left(\Delta E_{\mathrm{i}}\right)$ calculated for stigmasterol within the pre-polymerisation complex leading to a covalently produced cavity based on the deployment of 1,2 , or 3 free functional monomer mole equivalents. Also shown is the corresponding simulated energy $\left(\Delta E_{\mathrm{i}}\right)$ for the non-imprinted polymer (NIP) with 2 monomer mole equivalents of DMAAM. 
In the case of the non-imprinted polymer (NIP), no pre-association between the template and the monomers occurs and no pre-polymerisation template-monomer cluster complexes exist, with the consequence that the co-monomer molecules, e.g., DMAAM, can only initially interact with each other, and only after being polymerised (frozen) can the derived NIP polymer interact with stigmasterol in a non-specific fashion. For this reason, the $\Delta E_{\mathrm{i}}$ values for DMAAM in the MIP and NIP are different (Figure 1). These results indicated a clear preference in terms of the energetics of the binding interactions with the $N, N^{\prime}$-dimethylamino groups of the DMAAM monomers that were able to position around the template molecule, i.e., imprinted site, with the hybrid semi-covalent technique predicted to have the greatest differential between MIPs and NIPs at 2 mole equivalents of monomer.

\subsection{Preparation of the Molecularly Imprinted Polymers}

To confirm that the in silico modelling predictions associated with the use of a free functional co-monomer in combination with this semi-covalent imprinting approach resulted in a more effective MIP for the recognition of the stigmasterol, two MIP preparations, P1 and P2, respectively, were investigated, one with and the other without the functional co-monomer, $N, N^{\prime}$-DMAAM ( $2 \mathrm{mmol})$, present but with the cross-linker, ethyleneglycol dimethacrylate (EGDMA) (10 mmol), retained (Table 1$)$.

Table 1. Summary of the molecularly imprinted polymer (MIP), P1 and P2, and non-imprinted polymer (NIP), P3-P5, synthetic preparations used in this investigation ${ }^{1}$.

\begin{tabular}{cccccc}
\hline $\begin{array}{c}\text { Polymer } \\
\text { Code }\end{array}$ & $\begin{array}{c}\text { Template } \\
(\mathbf{m m o l})\end{array}$ & $\begin{array}{c}\text { DMAAM (FM) } \\
\mathbf{( m m o l )}\end{array}$ & $\begin{array}{c}\text { MAA (FM) } \\
\mathbf{( m m o l )}\end{array}$ & $\begin{array}{c}\text { EGDMA (Cross-Linker) } \\
\mathbf{( m m o l )}\end{array}$ & $\begin{array}{c}\text { Porogen } \\
\mathbf{( 6 \mathbf { m L } )}\end{array}$ \\
\hline P1 & 1 & 2 & - & 10 & $\mathrm{CHCl}_{3}$ \\
P2 & 1 & - & - & 10 & $\mathrm{CHCl}_{3}$ \\
P3 & - & 2 & - & 10 & $\mathrm{CHCl}_{3}$ \\
P4 & - & - & 2 & 10 & $\mathrm{CHCl}_{3}$ \\
P5 & - & - & - & 10 & $\mathrm{CHCl}_{3}$ \\
\hline
\end{tabular}

${ }^{1}$ All polymerisations were initiated with 2,2'-azobis(2-methyl-propionitrile) (AIBN) and performed at $60{ }^{\circ} \mathrm{C}$ for 24 h. FM: functional monomer.

Also, three different NIPs were prepared to examine the extent of non-specific binding that contributes to the overall interaction performance, and in particular the role that the functional and cross-linking monomers play with respect to these interactions. In each case, the non-imprinted polymers (NIPs) were prepared in the same manner as the imprinted polymers (MIPs) with the exception that the stigmasterol template $(1 \mathrm{mmol})$ was not included. For comparison, the non-imprinted polymer, P5, using only the crosslinker EGDMA was also included. To accommodate the possibility that non-specific interactions occur due to residual co-monomer functionalities residing within the binding cavity of the MIP post template extraction, NIPs were prepared with randomly dispersed MAA functional monomers (P4) or $N, N^{\prime}$-DMAAM monomers (P3). In this manner, it was possible to examine the extent to which these functionalities themselves in the absence of the stigmasterol imprinting template may contribute to non-specific adsorption.

\subsection{Evaluation of the Molecularly Imprinted Polymers}

Evaluation of the binding of stigmasterol to each of the imprinted and non-imprinted polymers was conducted via static batch binding methods with stigmasterol $(0.1 \mathrm{mM})$ dissolved in acetonitrile:water $(9: 1, v / v)$. These assays revealed that polymer P1, e.g., the hybrid 'semi-covalent MIP' containing $N, N^{\prime}$-DMAAM co-monomers, exhibited under these experimental conditions a greater binding capacity $\left(B_{P 1, \max }-B_{P 2, \max } \approx 8 \%\right)$ (Figure 2$)$ with an amount bound for the P1 MIP of ca. $5.6 \mathrm{mg}$ stigmasterol/g MIP polymer. Depending on the choice of co-monomer, differences in binding capacity and selectivity can be anticipated, which reflect subtle differences in the way the 
pre-polymerisation complex assembles with the same template. Clearly, not all of the observed binding of stigmasterol to P1 or P2 may have been due to the imprinting process per se as the non-imprinted polymers $\mathrm{P} 3$ and $\mathrm{P} 5$ also displayed some degree of binding. The binding to P5 represents the extent to which the cross-linker, poly-EGDMA, alone influences the overall binding, which, as expected, was found to be minimal $(<5 \%)$. The binding to the NIP P3, which was generated from the $N, N$ '-DMAAM co-monomer and the EGDMA cross-linker, represented a 'worst case scenario' with respect to non-specific binding. The binding result for P3 incorporates the extent to which non-specific interactions arise from DMAAM co-monomer units that were not involved in the formation of the pre-polymerization complex and therefore were not incorporated as part of the binding cavities, but instead were dispersed randomly throughout the polymer matrix. Similarly, the binding to P4 provides a useful insight into the possible interactions of stigmasterol, or indeed other sterol analytes, with randomly dispersed MAA functionalities rather than those solely localized within binding cavities. Although interactions of stigmasterol with MAA functionalities localized within binding cavities would be extremely unlikely due to the nature of the semi-covalent imprinting protocol employed in this investigation, it is interesting to note that the selective binding ( $\left.B_{\mathrm{MIP}}-B_{\mathrm{NIP}}\right)$ was statistically the same, regardless of whether the hybrid semi-covalent imprinting protocol or a semi-covalent imprinting protocol in the absence of $N, N^{\prime}$-DMAAM was employed with $17 \%\left(B_{\mathrm{P} 1, \max }-B_{\mathrm{P} 3, \max }\right)$ and $18 \%\left(B_{\mathrm{P} 2, \max }-B_{\mathrm{P} 5}\right.$, max $)$ selective binding, respectively. Since these values are based on 'the worst-case scenario' with respect to non-specific binding (i.e., binding of the template to the NIP P3), the actual selective binding and associated interaction affinity of the 'hybrid' adsorbent made by the semi-covalent approach will be higher.

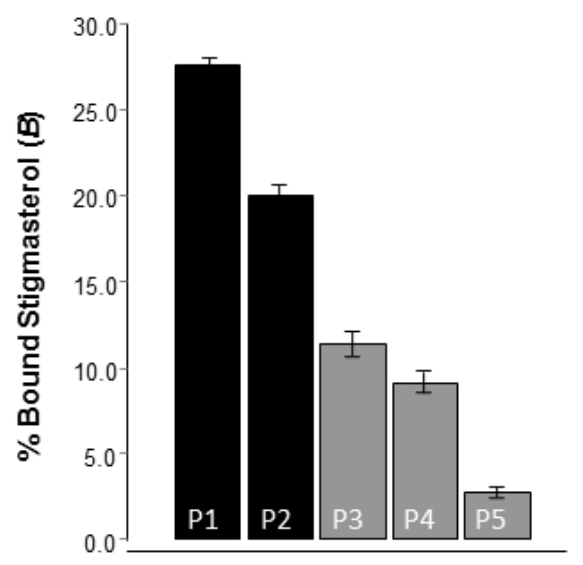

Figure 2. Stigmasterol binding evaluation for the imprinted (P1 and P2, black) and non-imprinted polymers (P3-P5, grey) under static batch binding conditions. Binding conditions are described in the Materials and Methods. The error bars indicate the standard error of the mean.

The ability of the stigmasterol-templated MIPs to recognize other $3 \beta$-hydroxy sterol analogues, such as ergosterol (a fungal sterol), cholesterol (an animal derived sterol), campesterol, and brassicasterol, was documented via single analyte binding and multiple analyte competitive binding studies. Since, in all cases, similar binding patterns were obtained with a higher binding capacity for the P1 MIP compared to the P2 MIP, only the data for the P1 MIP have been discussed below. The single analyte binding studies revealed that the P1 MIP preferentially 'recognized' each of these sterols with respect to the non-imprinted polymer P3 with imprinting factors of 4.0, 2.1, 5.1, 10.5, and 7.1, respectively (Figure 3), concordant with the A-ring C-3 $\beta$-hydroxyl group acting as a head group for binding via hydrogen bond interactions with the carboxyl and $N, N^{\prime}$-dimethylamido groups in the imprinted cavity of the MIP.

When the molecular structures of these phytosterols are taken into consideration, a rationale emerges with regard to the rank order of binding affinities observed for these compounds (Figure 4). 
Although only small variations occur in the structures of these sterols, the most prominent are associated with (a) the level of olefinic unsaturation at the $\Delta^{22,23}$ position; (b) the pattern of side chain substitution/stereochemistry of the D-ring alkyl chain tail groups, and (c) the extent of puckering of the $C$ and $D$ sterol rings due to the presence of the B-ring $\Delta^{5,6}$ double bond. Greater binding differences were apparent between brassicasterol and stigmasterol, which structurally differ by replacement of the $\mathrm{C}^{24 \mathrm{~S}}$-methyl group in brassicasterol by a $\mathrm{C}^{24 \mathrm{~S}}$-ethyl group in stigmasterol, than those that occurred between campesterol and stigmasterol, which structurally differ due to the replacement of the $\mathrm{C}^{24 \mathrm{~S}}$-ethyl substituent in stigmasterol by a $\mathrm{C}^{24 \mathrm{R}}$-methyl group as well as the lack of the $\Delta^{22,23}$ double bond in campesterol. Besides documenting the role that the single, common A-ring C-3 $\beta$-hydroxyl group plays in the molecular recognition of these phytosterols by the semi-covalently imprinted MIP, these results confirm the importance of shape determinants, mediated by van der Waals and Lifshitz force interactions, in determining the overall selectivity of these molecular imprinted polymers with these sterols.

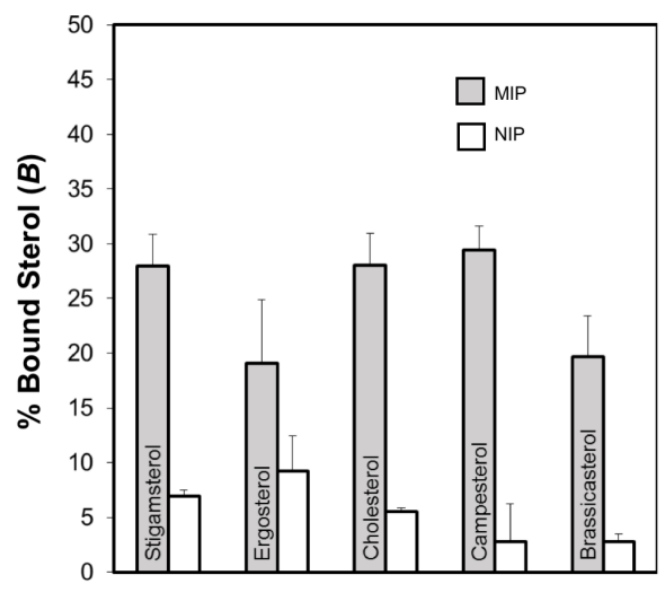

Figure 3. Static cross-reactivity binding evaluation based on single analyte binding studies with the semi-covalently imprinted stigmasterol MIP polymer P1 and the NIP polymer P3 with the sterols stigmasterol, ergosterol, cholesterol, campesterol, and brassicasterol. Binding conditions are described in the Materials and Methods. The error bars represent the standard error of the mean.

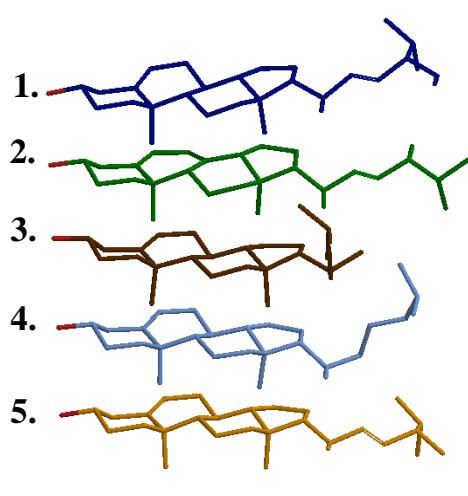

(a) side view

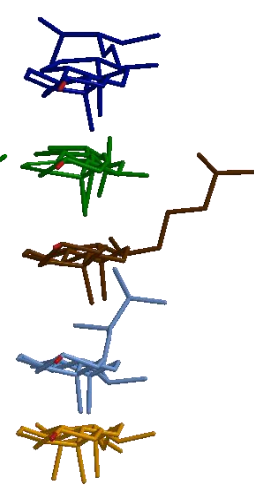

(b) end view

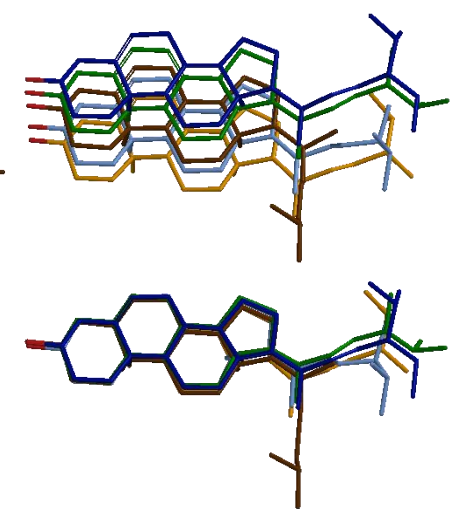

(c) top

view

(d) Offset

top view

Figure 4. Density functional equilibrium geometry models of five different sterol compounds shown in (a) side view; (b) end view; (c) top view; and (d) an offset top view, where the sterol compounds are: 1. stigmasterol, 2. ergosterol, 3. cholesterol, 4. campesterol, and 5. brassicasterol, illustrating their structural similarities and differences. All models were generated using SPARTAN ' 10 for Windows with the density functional equilibrium geometry calculation at the 3-21 G level. 


\section{Materials and Methods}

\subsection{LC Equipment and Methods}

An Agilent Technologies 1100 LC system (Waldbronn, Germany), consisting of a binary pump with vacuum degasser, an auto-sampler with a $900 \mu \mathrm{L}$ sample loop, and a thermostated column compartment, was employed for the analysis of the different sterol samples with an Agilent Technologies 1100 Series UV-Vis G1315C diode array detector SL (80 Hz) set to the absorbance wavelengths of 210, 280, and $330 \mathrm{~nm}$. Injected samples $(5 \mu \mathrm{L})$ were analysed by reversed-phase high-performance liquid chromatography (RP-HPLC) on a Zorbax Eclipse XDB-C 18 double end-capped column $\left(4.6 \times 150 \mathrm{~mm}, 5 \mu \mathrm{m}\right.$ particle size) (Agilent Technologies, Melbourne, Australia) at $23{ }^{\circ} \mathrm{C}$. Isocratic elution conditions were used with a mobile phase generated from a 5:95 ratio of solvent $\mathrm{A}$ $\left(0.1 \%(v / v) \mathrm{AcOH}\right.$ in $\left.\mathrm{H}_{2} \mathrm{O}\right)$ and solvent $\mathrm{B}(0.1 \%(v / v) \mathrm{AcOH}$ in $\mathrm{EtOH})$, respectively, at a flow rate of $0.5 \mathrm{~mL} \mathrm{~min}^{-1}$.

\subsection{Molecular Modelling}

All in silico modelling calculations were conducted using the Spartan '10 for Windows version 1.1.0 software package (Wavefunction, Inc., Irvine, CA, USA) on a Pentium IV 2.0 GHz computer. Modelling procedures were based on our previously described methods [29], whereby semi-empirical equilibrium geometry level theory was applied using a PM3 force field to calculate the heat of formation values $\left(\Delta H_{\mathrm{f}}\right)$ and the energy of interaction $\left(\Delta E_{\mathrm{i}}\right)$ for the template, the monomer clusters, and the template:monomer cluster complexes in the gas phase, initially without consideration of solvent effects. The impact of these solvent effects was then determined by inserting the template file into each monomer cluster file, without any pre-defined orientation imposed upon either the template or the monomer cluster, using an iterative approach. Moreover, using methodologies established in our previous modelling titration experiments, the template molecule stigmasterol was titrated in silico with the three commercially available functional monomers (FM) of different characteristics, namely, methacrylic acid (MAA), 4-vinylpyridine (4VP), and $N, N^{\prime}$-dimethylacrylamide $\left(N, N^{\prime}\right.$-DMAAM). A minimum of three iterations were carried out to derive the average net interaction energy $\left(\Delta E_{\mathrm{i}}\right)$ for the template:monomer cluster complex, as determined from Equation (1):

$$
\Delta E_{\mathrm{i}}=\Delta H_{\mathrm{f} \_ \text {template:monomer cluster complex }}-\left(\Delta H_{\mathrm{f} \_ \text {template }}+\Delta H_{\mathrm{f}_{-} \text {monomer cluster }}\right) \text {. }
$$

Modelling of the stigmasteryl methacrylate cavities (containing multiple equivalents of the free FM) post hydrolysis (i.e., after removal of the template) was achieved using the following procedure. Initially, as a first step, the stigmasteryl methacrylate template was modelled (using semi-empirical PM3 equilibrium geometry calculations with the MMFF) against increasing molar equivalents of the free functional monomer, e.g., MAA. The polymerisable groups were then 'frozen' (i.e., fixed in space with regard to all other adjacent atoms) to simulate the process of polymerisation, and semi-empirical PM3 equilibrium geometry calculations conducted again employing MMFF procedures. A virtual in silico 'base hydrolysis' was then performed, whereby the steryl portion of the template was removed, leaving the polymethacrylic acid functionalities (and additional free monomer units) within the cavity. As a second step, the polymerised methacrylic acid units were also 'frozen' to simulate the process of polymerisation using the MMFF procedures, permitting the free monomer units to be excluded and solvent included. Finally, as a third step, stigmasterol was then re-introduced into the cavity and semi-empirical PM3 equilibrium geometry calculations performed again, including all of the 'frozen' polymethacrylic acid atoms. The relevant average net interaction energy $\left(\Delta E_{\mathrm{i}}\right)$ for these MIP systems was determined from Equation (2):

$$
\Delta E_{\mathrm{i}}=\Delta H_{\mathrm{f} \_ \text {Binding complex }}-\left(\Delta H_{\mathrm{f}_{-} \text {target }}+\Delta H_{\mathrm{f} \_ \text {cavity }}\right)
$$


where $\Delta H_{\mathrm{f} \_ \text {Binding complex }}$ equates to the $\Delta H_{\mathrm{f}}$ of the complex between the cavity and the target (i.e., stigmasterol); $\Delta H_{\mathrm{f}_{-} \text {target }}$ equates to the $\Delta H_{\mathrm{f}}$ of the target; and $\Delta H_{\mathrm{f}_{-} \text {cavity }}$ equates to the $\Delta H_{\mathrm{f}}$ of the cavity generated by modeling the template (stigmasteryl methacrylate) in the presence of the free co-monomer/s (e.g., $N, N^{\prime}$-DMAAM).

An additional set of modelling experiments related to non-specific binding of the template was also undertaken to simulate the interaction with the non-imprinted polymer (NIP). This task was performed by modeling the monomer cluster (as done for the previous MIP modeling titrations) but in the absence of the template with the atoms of the monomers, e.g., acrylic acid groups, subsequently 'frozen' in place to simulate the process of polymerisation. The template file was then inserted into the monomer cluster file and the semi-empirical PM3 equilibrium geometry calculations again carried out starting from MMFF. The $\Delta E_{\mathrm{i}}$ values for this NIP systems were then calculated based on procedures similar to those described above for the MIP.

\subsection{Synthesis of Molecular Targets and Templates}

Cholesterol (Sigma-Aldrich, Sydney, Australia, 95\% purity), ergosterol (Fluka Chemicals, Melbourne, Australia, >95\% purity), and stigmasterol (Sigma Aldrich, Sydney, Australia and Acros Organics, NJ, USA, 95\% purity) were obtained from the vendors and their purity confirmed by RP-HPLC methods. Stigmasterol was converted in high yield to the reactive aldehyde (20S)-20-formyl-6 $\beta$-methoxy-3 $\alpha, 5$-cyclo-5 $\alpha$-pregnane (4) (Scheme 1).

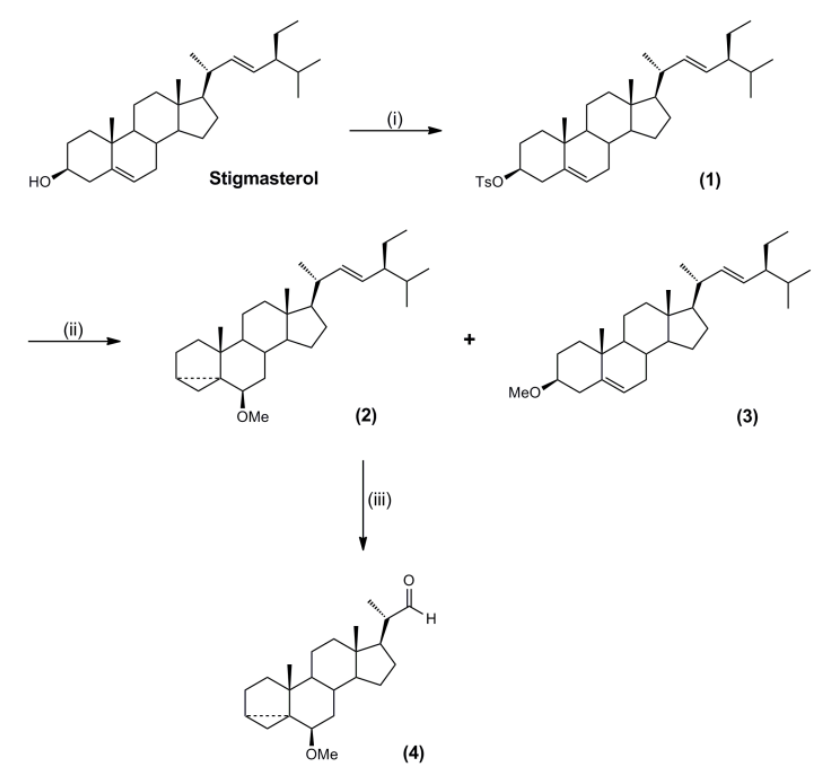

Scheme 1. Synthesis of (20S)-20-formyl-6 $\beta$-methoxy-3 $\alpha, 5$-cyclo-5 $\alpha$-pregnane (4) from stigmasterol. Reagents, conditions, and yield: (i) TsCl, pyridine, $22{ }^{\circ} \mathrm{C}, 72 \mathrm{~h}, 96 \%$; (ii) $\mathrm{MeOH}$, pyridine, reflux, $6 \mathrm{~h}$, $69 \%$; (iii) (a) $\mathrm{O}_{3}, \mathrm{DCM} /$ pyridine, $-78{ }^{\circ} \mathrm{C}$; (b) $\mathrm{Zn}, \mathrm{AcOH}, 22{ }^{\circ} \mathrm{C}, 3 \mathrm{~h}, \sim 100 \%$ crude. Nomenclature: stigmasteryl tosylate (1), (22E)-6 $\beta$-methoxy-3,5 $\alpha$-cyclo- $5 \alpha$-stigmasta-22-ene (stigmasterol $i$-methyl ether) (2), and 3-O-methylstigmasterol (3).

\subsection{Synthesis of Campesterol and Brassicasterol Targets}

The aldehyde (4) was then used as the synthetic precursor to prepare both campesterol (12) (Scheme 2) and brassicasterol (17) (Scheme 3) via strategies similar to those reported for the preparation of cholesterol and cholestadiene/cholestatriene derivatives [30-34]. Further experimental details related to the synthesis of the reactive intermediate (4) and its conversion to campersterol (12) and brassicasterol (17) are included in the Supplementary Materials section, please see S1: General experimental procedures and S2: Experimental details. 

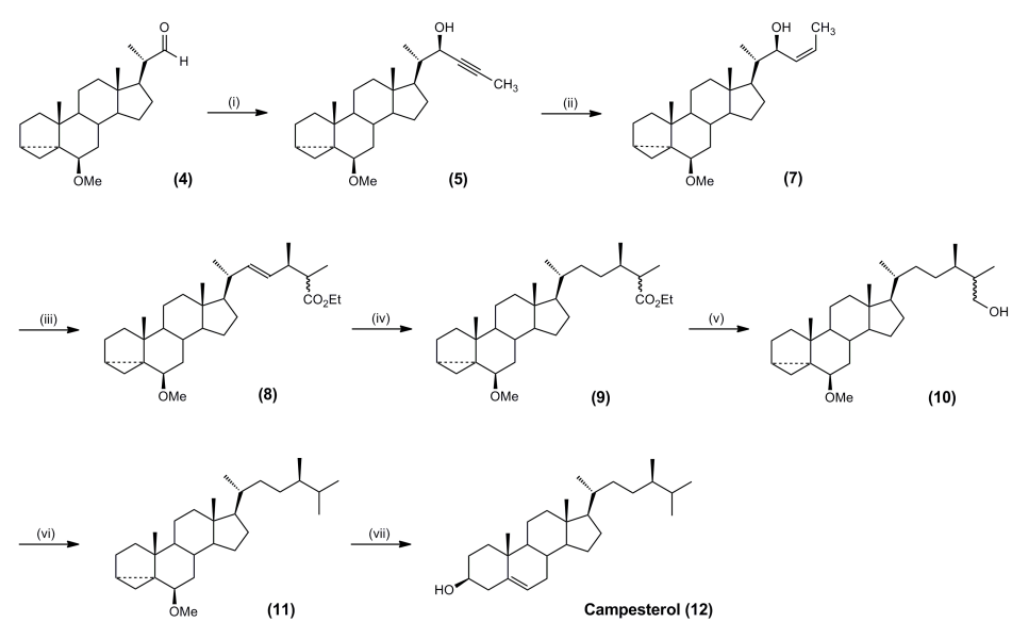

Scheme 2. Synthesis of campesterol (12) from (20S)-20-formyl-6 $\beta$-methoxy-3 $\alpha, 5$-cyclo- $5 \alpha$-pregnane (4). Reagents, conditions, and yield: (i) n-propynylmagnesium bromide, $\mathrm{THF}, 0{ }^{\circ} \mathrm{C}, 1 \mathrm{~h}, 17 \%$; (ii) $\mathrm{Pd} / \mathrm{CaCO}_{3}$, $\mathrm{Pb}$, quinoline, EtOAc, $\mathrm{H}_{2}, 3 \mathrm{~h}, 98 \%$; (iii) triethyl-orthopropionate, propionic acid, xylene, reflux, $1 \mathrm{~h}, ~ 100 \%$ crude; (iv) 10\% Pd/C, EtOAc, $\mathrm{H}_{2}, 20$ h, 96\%; (v) $\mathrm{LiAlH}_{4}, \mathrm{THF}, 22{ }^{\circ} \mathrm{C}, 2 \mathrm{~h}, 72 \%$; (vi) a. $\mathrm{MsCl}$, pyridine, $22{ }^{\circ} \mathrm{C}, 2 \mathrm{~h}, 94 \%$, b. $\mathrm{LiAlH}_{4}, \mathrm{THF}, 22{ }^{\circ} \mathrm{C}, 24 \mathrm{~h}, 94 \%$; (vii) $p$ - $\mathrm{TsOH}$, dioxane $/ \mathrm{H}_{2} \mathrm{O}$, reflux, $1 \mathrm{~h}, 87 \%$. Nomenclature: (22R)-6 $\beta$-methoxy-3 $\alpha, 5$-cyclo-26,27-dinor-5 $\alpha$-cholest-23-yn-22-ol (5), (22S)-6 $\beta$-methoxy-3 $\alpha, 5$-cyclo-26,27-dinor-5 $\alpha$-cholest-23-yn-22-ol (6), (22S,23Z)-6 $\beta$-methoxy-3 $\alpha$, 5-cyclo-26,27-dinor-5 $\alpha$-cholest-23-en-22-ol (7), (22E,24R)-ethyl-6 $\beta$-methoxy-24-methyl-3 $\alpha, 5$-cyclo- $5 \alpha$ cholest-22-en-26-oate (8), (24R)-ethyl-6 $\beta$-methoxy-24-methyl-3 $\alpha, 5$-cyclo-5 $\alpha$-cholestan-26-oate (9), (24R)-6 $\beta$-methoxy-24-methyl-3 $\alpha, 5$-cyclo-5 $\alpha$-cholestan-26-ol (10), and (24R)-6 $\beta$-methoxy-24-methyl-3 $\alpha$, 5-cyclo-5 $\alpha$-cholestane (campesterol $i$-methyl ether) (11).
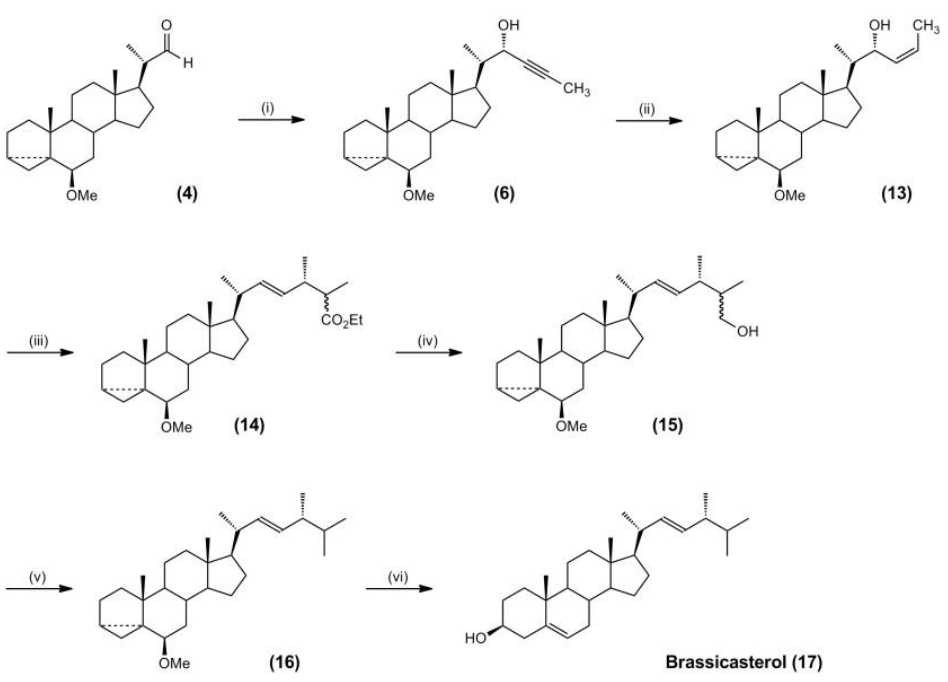

Scheme 3. Synthesis of brassicasterol (17) from (20S)-20-formyl-6 $\beta$-methoxy-3 $\alpha, 5$-cyclo- $5 \alpha$-pregnane (4). Reagents, conditions, and yield: (i) n-propynylmagnesium bromide, $\mathrm{THF}, 0{ }^{\circ} \mathrm{C}, 1 \mathrm{~h}, 13 \%$; (ii) $\mathrm{Pd} / \mathrm{CaCO}_{3}, \mathrm{~Pb}$, quinoline, $\mathrm{H}_{2}$, EtOAc, $3 \mathrm{~h}, 99 \%$; (iii) triethylorthopropionate, propionic acid, xylene, reflux, $1 \mathrm{~h}, 100 \%$ crude; (iv) $\mathrm{LiAlH}_{4}$, THF, $22{ }^{\circ} \mathrm{C}, 2 \mathrm{~h}, 98 \%$; (v) (a) $\mathrm{MsCl}$, pyridine, $22{ }^{\circ} \mathrm{C}$, $2 \mathrm{~h}, 100 \%$ crude, (b) $\mathrm{LiAlH}_{4}, \mathrm{THF}, 22{ }^{\circ} \mathrm{C}, 21 \mathrm{~h}, 100 \%$ crude; (vi) $p$-TsOH, dioxane $/ \mathrm{H}_{2} \mathrm{O}$, reflux, 1 h, 73\%. Nomenclature: (22S)-6 $\beta$-methoxy-3 $\alpha, 5$-cyclo-26,27-dinor-5 $\alpha$-cholest-23-yn-22-ol (6), $(22 R, 23 Z)-6 \beta$-methoxy-3 $\alpha, 5$-cyclo-26,27-dinor-5 $\alpha$-cholest-23-en-22-ol (13), (22E,24S)-ethyl-6 $\beta$-methoxy24-methyl-3 $\alpha, 5$-cyclo-5 $\alpha$-cholest-22-en-26-oate (14), (22E,24S)-6 $\beta$-methoxy-24-methyl-3 $\alpha, 5$-cyclo- $5 \alpha$-cholest -22-en-26-ol (15), and (22E,24R)-6 $\beta$-methoxy-24-methyl-3 $\alpha, 5$-cyclo-5 $\alpha$-cholest-22-ene (brassicasterol i-methyl ether) (16). 


\subsection{Synthesis of the Polymerisable Molecular Template}

Stigmasterol was converted to the polymerisable template stigmasteryl methacrylate (18) via a dicyclohexylcarbodiimide (DCC)-mediated reaction [28] between stigmasterol and methacrylic acid (Scheme 4) based on conditions similar to those used for generation of cholesterol-based templates [15]. Further details of the synthesis of (18) are included in the Supplementary Materials section, Figures S1 and S2.
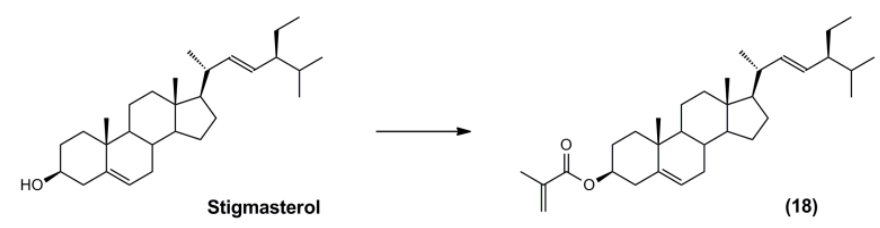

Scheme 4. The preparation of the stigmasteryl methacrylate (18). Reagents, conditions, and yield:

(i) DCC, 4-(dimethylamino)pyridine (DMAP), dichloromethane (DCM), room temp, $18 \mathrm{~h}, 74 \%$.

\subsection{MIP Preparation}

Stigmasteryl methacrylate $(0.480 \mathrm{~g}, 1 \mathrm{mmol}), N, N^{\prime}$-DMAAM $(206 \mu \mathrm{L}, 2 \mathrm{mmol})$, and $\mathrm{CHCl}_{3}(6 \mathrm{~mL})$ were placed in $15 \mathrm{~mL}$ test tubes fitted with a suba seal. The mixture was cooled on ice, purged with $\mathrm{N}_{2 \text { (gas) }}$ for $2 \mathrm{~min}$, and left on ice for a further $28 \mathrm{~min}$. Ethyleneglycol dimethacrylate (EGDMA) $(1.89 \mathrm{~mL}$, $10 \mathrm{mmol}$ ) and 2,2'-azo-bis(2-methyl-propionitrile) (AIBN) $(50 \mathrm{mg}, 0.31 \mathrm{mmol}$ ) were then added. The mixture was again purged with $\mathrm{N}_{2 \text { (gas) }}$ for a further $5 \mathrm{~min}$. This pre-polymerisation mixture was placed in a thermostated water bath at $60^{\circ} \mathrm{C}$ for $24 \mathrm{~h}$. A non-imprinted polymer (NIP) was prepared in a similar manner with the exception that the polymerisable template (stigmasteryl methacrylate) was not included. Additional non-imprinted polymers were prepared without stigmasteryl methacrylate or a $N, N^{\prime}$-DMAAM functional monomer; however, MAA $(84.4 \mu \mathrm{L}, 1 \mathrm{mmol})$ was included in the pre-polymerisation mixture. The polymers were removed from the reaction tubes, then crushed and ground using a Retsch PM 200 ball mill (Haan, Germany) operated at $300 \mathrm{rpm}$ for $6 \mathrm{~min}$ in intervals of 2 min bursts. The template was removed from the MIP resin by base hydrolysis in methanolic $\mathrm{NaOH}$ $(1.5 \mathrm{M}, 150 \mathrm{~mL})$ under reflux overnight. The MIP was then extensively extracted with methanol via Soxhlet procedures, filtered, and dried in vacuo. A summary of various conditions used for the MIP preparation, schematically shown in Scheme 5, and the NIP is given in Table 1.
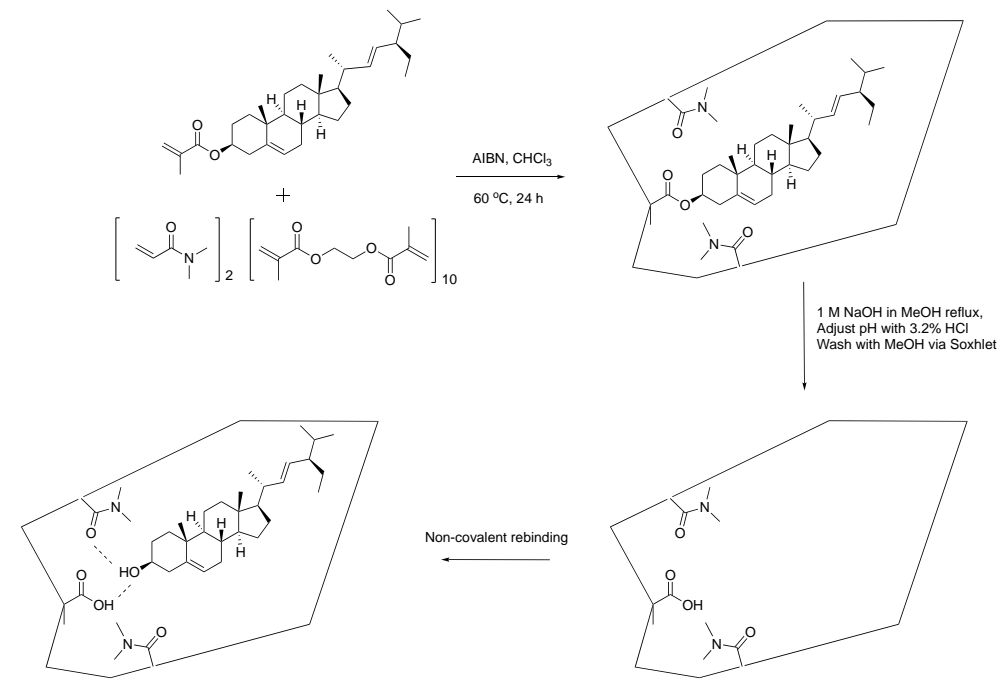

Scheme 5. The preparation of a hybrid semi-covalent MIP for stigmasterol using the stigmasterol methacrylate template (18) and the $N, N^{\prime}$-DMAAM co-monomer. 


\subsection{MIP Batch Binding Evaluation}

Batch binding studies were conducted in triplicate to evaluate the binding attributes of the stigmasterol-imprinted polymer and the non-imprinted polymer using stigmasterol $(0.1 \mathrm{mM})$ in acetonitrile $/ \mathrm{H}_{2} \mathrm{O}(9: 1 \mathrm{v} / \mathrm{v})$. The MIP and NIP polymers $(20 \mathrm{mg})$ were weighed into sealable Eppendorf tubes $(1.7 \mathrm{~mL})$ and incubated at $20{ }^{\circ} \mathrm{C}$ with the analyte solution $(1.0 \mathrm{~mL}, 0.1 \mathrm{mM})$ on a rotary mixer spinning at $40 \mathrm{rpm}$ for $18 \mathrm{~h}$. The mixture was then centrifuged at $16,060 \times \mathrm{g}$ for $30 \mathrm{~min}$ to pellet the analyte-bound polymer complex. An aliquot $(100 \mu \mathrm{L})$ of the supernatant was removed and analysed by RP-HPLC with UV detection at $210 \mathrm{~nm}$ and $280 \mathrm{~nm}$. The amount of analyte bound (B) was determined as the difference between the initial analyte concentration and the test concentration. Cross-reactivity studies using solutions $(0.1 \mathrm{mM})$ of ergosterol, cholesterol, campesterol, or brassicasterol in acetonitrile $/ \mathrm{H}_{2} \mathrm{O}(9: 1 \mathrm{v} / \mathrm{v})$ were also performed in a similar manner.

\section{Conclusions}

In this investigation, several molecularly imprinted polymers were designed and prepared using the template stigmasteryl methacrylate (18) via the semi-covalent-imprinting approach. The derived MIPs were found to efficiently bind stigmasterol and several other sterol targets. The advantages of utilising design criteria based on molecular modelling procedures and interaction energy calculations to guide the selection of the monomer type, as well as the choice of the monomer:template ratios for the formation of the pre-polymerisation complex with these semi-covalently imprinted polymers, was documented. The formation of a stable pre-polymerization complex requires a close proximity of template and functional monomer. This can be achieved through the interplay of hydrogen bonding between the template and the monomer if that is possible, but other effects (electrostatic, van derWaals, Lipshitz, dipolar, etc.) can also participate. What the current investigation demonstrates is that at the stage of formation of the pre-polymerization complex, hydrogen bond interactions are not required under covalent imprinting conditions to form a stable complex. However, post cleavage and removal of the stigmasterol template, hydrogen bond capabilities are regenerated in the MIP cavity, and hydrogen bond interactions with the 3-hydroxy group of the sterol can then occur. Moreover, the importance of shape determinants in controlling the binding selectivity with templates that contain only a single hydrogen bonding site was established. Collectively, these results have provided additional insights into the unique binding propensities of molecularly imprinted polymers which involve only a single hydrogen bonding site, but are capable of molecular recognition through shape, van der Waals, and non-polar Lifshitz force interactions. In addition, these investigations have provided a basis for our ongoing research into the use of MIP separation technologies with a focus on the development of alternative routes for the recovery of phytosterols from plant waste sources, compared to current techniques involving protracted extraction procedures or costly synthetic methods.

Supplementary Materials: The following are available online at http:/ / www.mdpi.com/2311-5629/4/1/13/s1, S1: General experimental procedures, S2: experimental details, Figure S1: ${ }^{1} \mathrm{H}$ NMR $\left(400 \mathrm{MHz}, \mathrm{CDCl}_{3}\right)$ of stigmasteryl methacrylate (18), Figure S2: ${ }^{13} \mathrm{C} \mathrm{NMR}\left(100 \mathrm{MHz}, \mathrm{CDCl}_{3}\right)$ of stigmasteryl methacrylate (18).

Acknowledgments: The authors gratefully acknowledge financial support from the CSIRO National Research Food Futures Flagship.

Author Contributions: L.J.S., B.D., and M.T.W.H. conceived and designed the experiments; L.J.S., B.K.Y.L., and B.D. performed the experiments; L.J.S., S.J.H., R.I.B., and M.T.W.H. analyzed the data; B.D. and R.I.B. contributed reagents/materials/analysis tools; L.J.S., R.I.B., and M.T.W.H. wrote the paper.

Conflicts of Interest: The authors declare no conflict of interest. 


\section{References}

1. Abidi, S.L. Chromatographic analysis of plant sterols in foods and vegetable oils. J. Chromatogr. A 2001, 935, 173-201. [CrossRef]

2. Fernandes, P.; Cabral, J.M.S. Phytosterols: Applications and recovery methods. Bioresour. Technol. 2007, 98, 2335-2350. [CrossRef] [PubMed]

3. Schröder, M.; Vetter, W. High-speed counter-current chromatographic separation of phytosterols. Anal. Bioanal. Chem. 2011, 400, 3615-3623. [CrossRef] [PubMed]

4. Yan, F.; Yang, H.; Li, J.; Wang, H. Optimization of phytosterols recovery from soybean oil deodorizer distillate. J. Am. Oil Chem. Soc. 2012, 89, 1363-1370. [CrossRef]

5. Rosello-Soto, E.; Koubaa, M.; Moubarik, A.; Lopes, R.P.; Saraiva, J.A.; Boussetta, N.; Grimi, N.; Barba, F.J. Emerging opportunities for the effective valorization of wastes and by-products generated during olive oil production process: Non-conventional methods for the recovery of high-added value compounds. Trends Food Sci. Technol. 2015, 45, 296-310. [CrossRef]

6. Uddin, M.S.; Sarker, M.Z.I.; Ferdosh, S.; Akanda, M.J.H.; Easmin, S.; Bt Shamsudin, S.H.; Yunus, K.B. Phytosterols and their extraction from various plant matrices using supercritical carbon dioxide: A review. J. Sci. Food Agric. 2015, 95, 1385-1394. [CrossRef] [PubMed]

7. Cheong, W.J.; Yang, S.H.; Ali, F. Molecular imprinted polymers for separation science: A review of reviews. J. Sep. Sci. 2013, 36, 609-628. [CrossRef] [PubMed]

8. Schwarz, L.J.; Danylec, B.; Harris, S.J.; Boysen, R.I.; Hearn, M.T.W. Sequential molecularly imprinted solid-phase extraction methods for the analysis of resveratrol and other polyphenols. J. Chromatogr. A 2016, 1438, 22-30. [CrossRef] [PubMed]

9. Hashim, S.N.N.S.; Schwarz, L.J.; Danylec, B.; Mitri, K.; Yang, Y.; Boysen, R.I.; Hearn, M.T.W. Recovery of ergosterol from the medicinal mushroom, Ganoderma tsugae var. Janniae, with a molecularly imprinted polymer derived from a cleavable monomer-template composite. J. Chromatogr. A 2016, 1468, 1-9. [CrossRef] [PubMed]

10. Danylec, B.; Schwarz, L.J.; Harris, S.J.; Boysen, R.I.; Hearn, M.T.W. The application of template selectophores for the preparation of molecularly imprinted polymers. Molecules 2015, 20, 17601-17613. [CrossRef] [PubMed]

11. Dickert, F.L.; Hayden, O. Molecular imprinting in chemical sensing. TrAC Trends Anal. Chem. 1999, 18, 192-199. [CrossRef]

12. Whitcombe, M.J.; Kirsch, N.; Nicholls, I.A. Molecular imprinting science and technology: A survey of the literature for the years 2004-2011. J. Mol. Recognit. 2014, 27, 297-401. [PubMed]

13. Boysen, R.I.; Schwarz, L.J.; Nicolau, D.V.; Hearn, M.T.W. Molecularly imprinted polymer membranes and thin films for the separation and sensing of biomacromolecules. J. Sep. Sci. 2017, 40, 314-335. [CrossRef] [PubMed]

14. Whitcombe, M.J.; Rodriguez, M.E.; Villar, P.; Vulfson, E.N. A new method for the introduction of recognition site functionality into polymers prepared by molecular imprinting: Synthesis and characterisation of polymeric receptors for cholesterol. J. Am. Chem. Soc. 1995, 117, 7105-7111. [CrossRef]

15. Sellergren, B.; Wieschemeyer, J.; Boos, K.S.; Seidel, D. Imprinted polymers for selective adsorption of cholesterol from gastrointestinal fluids. Chem. Mater. 1998, 10, 4037-4046. [CrossRef]

16. Kim, J.M.; Ahn, K.D.; Wulff, G. Cholesterol esterase activity of a molecularly imprinted polymer. Macromol. Chem. Phys. 2001, 202, 1105-1108. [CrossRef]

17. Hwang, C.C.; Lee, W.C. Chromatographic characteristics of cholesterol-imprinted polymers prepared by covalent and non-covalent imprinting methods. J. Chromatogr. A 2002, 962, 69-78. [CrossRef]

18. Gore, M.A.; Karmalkar, R.N.; Kulkarni, M.G. Enhanced capacities and selectivities for cholesterol in aqueous media by molecular imprinting: Role of novel cross-linkers. J. Chromatogr. B 2004, 804, 211-221. [CrossRef] [PubMed]

19. Ciardelli, G.; Borrelli, C.; Silvestri, D.; Cristallini, C.; Barbani, N.; Giusti, P. Supported imprinted nanospheres for the selective recognition of cholesterol. Biosens. Bioelectron. 2006, 21, 2329-2338. [CrossRef] [PubMed]

20. Puoci, F.; Curcio, M.; Cirillo, G.; Iemma, F.; Spizzirri, U.G.; Picci, N. Molecularly imprinted solid-phase extraction for cholesterol determination in cheese products. Food Chem. 2008, 106, 836-842. [CrossRef] 
21. Aghaei, A.; Milani Hosseini, M.R.; Najafi, M. A novel capacitive biosensor for cholesterol assay that uses an electropolymerized molecularly imprinted polymer. Electrochim. Acta 2010, 55, 1503-1508. [CrossRef]

22. Kitahara, K.I.; Yoshihama, I.; Hanada, T.; Kokuba, H.; Arai, S. Synthesis of monodispersed molecularly imprinted polymer particles for high-performance liquid chromatographic separation of cholesterol using templating polymerization in porous silica gel bound with cholesterol molecules on its surface. J. Chromatogr. A 2010, 1217, 7249-7254. [CrossRef] [PubMed]

23. Li, J.; Zhang, Z.; Xu, S.; Chen, L.; Zhou, N.; Xiong, H.; Peng, H. Label-free colorimetric detection of trace cholesterol based on molecularly imprinted photonic hydrogels. J. Mater. Chem. 2011, 21, 19267-19274. [CrossRef]

24. Ye, L.; Weiss, R.; Mosbach, K. Synthesis and characterization of molecularly imprinted microspheres. Macromolecules 2000, 33, 8239-8245. [CrossRef]

25. Ye, L.; Yu, Y.H.; Mosbach, K. Towards the development of molecularly imprinted artificial receptors for the screening of estrogenic chemicals. Analyst 2001, 126, 760-765. [CrossRef] [PubMed]

26. Szumski, M.; Buszewski, B. Molecularly imprinted polymers: A new tool for separation of steroid isomers. J. Sep. Sci. 2004, 27, 837-842. [CrossRef] [PubMed]

27. Zhu, Q.; Wang, L.; Wu, S.; Joseph, W.; Gu, X.; Tang, J. Selectivity of molecularly imprinted solid phase extraction for sterol compounds. Food Chem. 2009, 113, 608-615. [CrossRef]

28. Hashim, S.N.N.S.; Boysen, R.I.; Schwarz, L.J.; Danylec, B.; Hearn, M.T.W. A comparison of covalent and non-covalent imprinting strategies for the synthesis of stigmasterol imprinted polymers. J. Chromatogr. A 2014, 1359, 35-43. [CrossRef] [PubMed]

29. Boysen, R.I.; Schwarz, L.J.; Li, S.; Chowdhury, J.; Hearn, M.T.W. Photolithographic patterning of biomimetic molecularly imprinted polymer thin films onto silicon wafers. Microsyst. Technol. 2014, 20, 2037-2043. [CrossRef]

30. Chung, S.K.; Ryoo, C.H.; Yang, H.W.; Shim, J.Y.; Kang, M.G.; Lee, K.W.; Kang, H.I. Synthesis and bioactivities of steroid derivatives as antifungal agents. Tetrahedron 1998, 54, 15899-15914. [CrossRef]

31. Fujimoto, Y.; Kimura, M.; Khalifa, F.A.M.; Ikekawa, N. Side chain structural requirement for utilization of sterols by the silkworm for growth and development. Non-stereoselective utilization of the 24-stereoisomeric pairs of 24-alkylsterols. Chem. Pharm. Bull. 1984, 32, 4372-4381. [CrossRef]

32. Hutchins, R.F.N.; Thompson, M.J.; Svoboda, J.A. The synthesis and the mass and nuclear magnetic resonance spectra of side chain isomers of cholesta-5,22-dien-3 $\beta$-ol and cholesta-5,22,24-trien-3 $\beta$-ol. Steroids 1970, 15, 113-130. [CrossRef]

33. Partridge, J.J.; Faber, S.; Uskoković, M.R. Vitamin D3 metabolites I. Synthesis of 25-hydroxycholesterol. Helv. Chim. Acta 1974, 57, 764-771. [CrossRef] [PubMed]

34. Steele, J.A.; Mosettig, E. The solvolysis of stigmasteryl tosylate. J. Org. Chem. 1963, 28, 571-572. [CrossRef] 